\title{
Probing SH2-domains using Inhibitor Affinity Purification (IAP)
}

\author{
Michael Höfener ${ }^{1 \dagger}$, Stephanie Heinzlmeir ${ }^{2,3,4 \dagger}$, Bernhard Kuster ${ }^{2,5}$ and Norbert Sewald ${ }^{1 *}$
}

\begin{abstract}
Background: Many human diseases are correlated with the dysregulation of signal transduction processes. One of the most important protein interaction domains in the context of signal transduction is the Src homology 2 (SH2) domain that binds phosphotyrosine residues. Hence, appropriate methods for the investigation of $\mathrm{SH} 2$ proteins are indispensable in diagnostics and medicinal chemistry. Therefore, an affinity resin for the enrichment of all SH2 proteins in one experiment would be desirable. However, current methods are unable to address all $\mathrm{SH} 2$ proteins simultaneously with a single compound or a small array of compounds.

Results: In order to overcome these limitations for the investigation of this particular protein family in future experiments, a dipeptide-derived probe has been designed, synthesized and evaluated. This probe successfully enriched $22 \mathrm{SH} 2$ proteins from mixed cell lysates which contained $50 \mathrm{SH} 2$ proteins. Further characterization of the $\mathrm{SH} 2$ binding properties of the probe using depletion and competition experiments indicated its ability to enrich complexes consisting of $\mathrm{SH} 2$ domain bearing regulatory PI3K subunits and catalytic phosphoinositide 3-kinase (PI3K) subunits that have no $\mathrm{SH} 2$ domain.

Conclusion: The results make this probe a promising starting point for the development of a mixed affinity resin with complete $\mathrm{SH} 2$ protein coverage. Moreover, the additional findings render it a valuable tool for the evaluation of PI3K complex interrupting inhibitors.
\end{abstract}

Keywords: Chemical proteomics, Inhibitor affinity purification, Mass spectrometry, PI3 kinase, SH2 domain

\section{Background}

Many human diseases are caused by imbalanced regulation of crucial cellular processes. Cell metabolism is regulated via a multitude of complex signal transduction cascades. Signals derived from extracellular stimuli are forwarded by phosphorylation events, which need to be strictly controlled. Dysregulation of these signaling processes can cause many human diseases such as cancer [1]. Different protein kinases are key regulators of the corresponding regulation cascades and, therefore, one of the most prominent research targets in drug development $[2,3]$. In the initial activation events many protein complexes are formed in order to initiate phosphorylation cascades that further activate transcription factors. Hence, the investigation of these complexes is of major

\footnotetext{
*Correspondence: norbert.sewald@uni-bielefeld.de

${ }^{\dagger}$ Equal contributors

'Organic and Bioorganic Chemistry, Department of Chemistry, Bielefeld

University, Universitätsstraße 25, 33615 Bielefeld, Germany

Full list of author information is available at the end of the article
}

importance and still remains challenging since the participating proteins are usually expressed at low levels $[4,5]$. There are many examples in the literature of kinase inhibitors that were applied in clinical studies or are available as approved drugs [6]. These kinase inhibitors usually address the ATP binding domain in the active (type 1 inhibitors) or inactive (type 2 inhibitors) conformation of the enzyme. The prohibition of protein interactions required for activation of signal transduction kinases is an alternative strategy for the direct inhibition. The SH2 (Src homology 2) domain that binds to phosphorylated tyrosine $(\mathrm{pY})$ residues is one of the most important domains responsible for protein interaction. $120 \mathrm{SH} 2$ domains are present in 110 distinct human proteins according to the UniProt database and Liu et al. [7-9]. $\mathrm{SH} 2$ domain mediated PI3K heterodimer formation is a prominent example of such a protein interaction. PI3 kinases participate in signal transduction by generating second messengers that further activate multiple effector pathways, including Akt-, NF-kB- and Jnk-signaling. PI3 
kinases are correlated with various diseases such as cancer [10]. Taking this aspect into account, it is not surprising that addressing the $\mathrm{SH} 2$ binding domain is an important strategy for therapeutic purposes [11]. A mixed kinase affinity resin for the evaluation of ATP competitive kinase inhibitors, called Kinobeads [12] is available and a comparable affinity-based approach for the evaluation of $\mathrm{SH} 2$ addressing inhibitors would be desirable. Kinobeads consist of different affinity probes immobilized on a solid phase and have been proven to capture a major part of the human kinome by addressing the ATP binding pocket. They are used to investigate the kinase target spectrum and for the determination of binding affinities in competition experiments with ATP competitive drugs [12]. A mixed SH2 domain affinity resin that addresses the majority of $\mathrm{SH} 2$ containing proteins would be desirable to adopt this methodology to $\mathrm{SH} 2$ proteins. Methods based on a variety of bait peptides (e.g. pY-peptide chips) have already been developed for the investigation of these protein-protein interactions $[13,14]$. However, coverage of the full range of SH2proteins turned out to be problematic. $70 \mathrm{SH} 2$ proteins could be addressed by utilizing a mixture of 6200 different immobilized 13-membered pY-peptides [14]. One disadvantage of this chip-based method is the requirement of SH2-GST fusion proteins for the analysis of the profiling experiments via anti-GST fluorescent antibodies. An inhibitor affinity purification (IAP) based approach was established for the investigation of the EGFR interactome that used at least 24 different immobilized pY-peptides for probing SH2 proteins $[15,16]$. The IAP approach with the highest amount of captured SH2 proteins used 57 13-membered pY-peptides immobilized on magnetic beads that captured $45 \mathrm{SH} 2$ proteins [14]. However, the number of compounds immobilized in the IAP experiment inversely correlates with the effective concentration of each immobilized substance [17]. As a result low abundant $\mathrm{SH} 2$ protein members will not be enriched sufficiently. Hence, all described efforts to develop an IAP approach that covers all 110 human $\mathrm{SH} 2$ proteins were not successful. In this study a small pY containing dipeptide that only addresses the conserved pY-core motif addressing the $\mathrm{SH} 2$ domain was used as a probe for the IAP experiment to potentially overcome these limitations in the future.

\section{Results/discussion}

\section{Design and synthesis}

For the IAP approach a linkable probe that carries a SH2 domain affinity group and allows for the immobilization on a solid support is required (Figure 1) [19]. SH2 domains recognize pY-peptides of at least three to eight residues and consist of two major binding pockets, the highly conserved pY pocket and the hydrophobic $\mathrm{pY}+3$ pocket [20]. Both regions were shown to be essential for SH2 ligand binding and are, therefore, highly conserved [21]. The two point interaction mode induced by the conserved $\mathrm{pY}$ and $\mathrm{pY}+3$ pocket is also known as "two-pronged plug two-holded socket" binding model [11]. In addition to the conserved binding regions further binding pockets are mainly responsible for the specificity of the ligand recognition. Hence, for the development of specific ligands such additional pockets are often addressed e.g. pY-2 to pY+3 (VIpYFVP) or pY-1 to pY+5 (GPYLPQTV) [22]. In addition, cyclic peptidomimetics that are less flexible have been developed for selective inhibition of distinct $\mathrm{SH} 2$ proteins [23]. In order to create a $\mathrm{SH} 2$ protein probe that is potent towards $\mathrm{SH} 2$ proteins but not selective for a specific protein a small peptide that only addresses $\mathrm{pY}$ to $\mathrm{pY}+3$ seems appropriate for the inhibitor moiety. All natural $\mathrm{SH} 2$ ligands contain a $\mathrm{pY}$ residue and it was shown that the omission of the phosphate group decreased the binding affinity towards $\mathrm{SH} 2$ domains significantly. In general, a pY residue is essential for a potent probe and, therefore, has to be incorporated into the probe structure. Glutamic acid in $\mathrm{pY}+1$ position is known to have a flexible binding pattern [21]

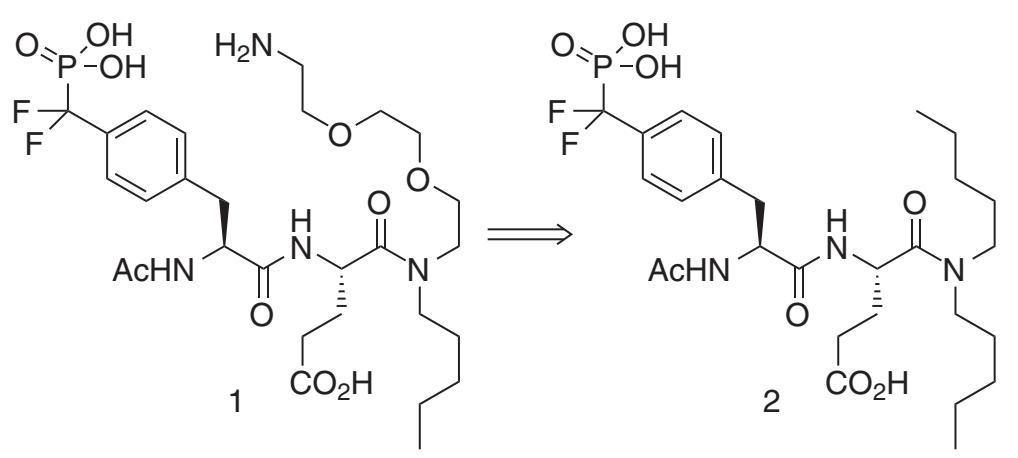

Figure 1 Probe design. Probe $\mathbf{1}$ is based on the peptide inhibitor $\mathbf{2}$ [18]. It can be immobilized on a solid support via the primary amine function of its linker moiety. 
and, as a result, seems to be a good residue for a mixed $\mathrm{SH} 2$-protein affinity resin. N-Acetylated phosphotyrosine residues are present in many $\mathrm{SH} 2$ inhibitors and, therefore, may be considered as potential moiety for the probe structure. The $\mathrm{SH} 2$ domain inhibitor 2 that was previously applied for a thermodynamic and structural study on SH2 domains [18] was chosen as starting point for the probe development, since it consists of several key moieties for a potent $\mathrm{SH} 2$ domain probe with broad SH2 protein affinity. Additionally, X-ray crystal structures of $\mathrm{SH} 2$ proteins co-crystallized with inhibitor $\mathbf{2}$ are available to determine potential linker modification sites. Another key feature of inhibitor $\mathbf{2}$ is its non-hydrolysable phosphotyrosine building block that was incorporated into the molecule without significant loss of biological activity. One of the inhibitor's pentyl residues was chosen for linker modification, since it points outside the binding site. Molecular docking analysis was used to confirm similar target binding properties of probe 1 compared to inhibitor 2 [18]. The binding mode of the modified pYE SH2 ligand $\mathbf{1}$ (probe $\mathbf{1}$ ) is in accordance with the original inhibitor 2 (Figure 2) [24]. Due to the chosen linker modification the $\mathrm{pY}+2$ is rationally not addressed by the probe, but the attached alkyl chain addresses the $\mathrm{pY}+3$ binding pocket. For the alkyl chain that addresses the $\mathrm{pY}+3$ pocket, the small flexible n-pentyl chain as it can be found in inhibitor 2 , was kept in the probe molecule in order to cover a broad spectrum of $\mathrm{SH} 2$ proteins [25]. However, the results of the docking experiments revealed that the $\mathrm{pY}$ +2 pocket may be occupied by the oligoethyleneglycol linker (Figure 2). These findings indicate that probe $\mathbf{1}$ is a promising candidate for the IAP approach. As shown in Figure 2, the phosphonic acid is mainly stabilized by electrostatic interactions with the side chains of R158,
R178, S180, T182 and T183 and the amide protons of R158, E181, T182 and T183 (amino acid positions adopted from PDB: 1a08). The aryl moiety of the pY residue is stabilized via cation- $\pi$-interactions with R158 and R178. The carbonyl groups of the $\mathrm{N}$-acetyl group, the pY backbone and the glutamic acid side chain interact with H204. In addition, the carbonyl group of the glutamic acid side chain is also stabilized by electrostatic interactions with Y205. The pentyl residue binds to a hydrophobic pocket formed by Y205, I217, T218 and L240.

According to the synthesis described by $\mathrm{Vu}$ et al. [5], probe 1 can easily be obtained using manual solid phase peptide synthesis on a 2-chlorotrityl resin. The required F2pmp building block was directly synthesized by copper mediated cross coupling of an organozinc compound in analogy to published procedures [26-31].

\section{SH2 enrichment}

The probe was immobilized on sepharose beads and pull-down experiments were performed using mixed cell lysates (Colo205, K562, Ovcar8 and SKNBE2). Since the utilized cell lines feature different protein profiles, a mix of these lysates offers a versatile pool of proteins for pull-down experiments. This cell lysate mix was used for protein kinase enrichment before and performed well, which led to the conclusion, that it might also contain a high variety of other signaling-related proteins such as SH2 proteins. Therefore, this mixed cell lysate was chosen for the first pull-down experiments (Additional file 1). Captured proteins were eluted from the beads, digested with trypsin and identified using liquid chromatography tandem mass spectrometry (LC-MS/MS). This analysis gave 1557 proteins in total, including 22 SH2proteins. SH2 proteins have been enriched with an

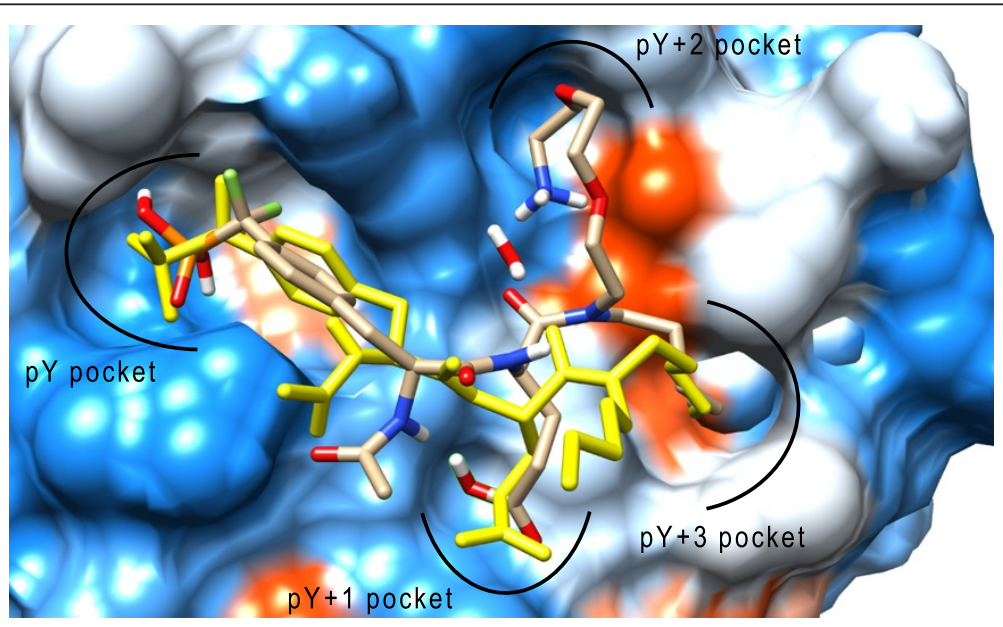

Figure 2 Target binding properties of probe 1. X-ray crystal structure of the pYE SH2 ligand 2 [18] (yellow) in complex with the c-Src-SH2 domain (PDB: 1a08). Probe $\mathbf{1}$ was docked to that protein structure and the docking pose incorporated into that complex (atom specific coloring). 
intensity ratio of $2.7 \%$, using summed intensities for $\mathrm{SH} 2$ proteins compared to the summed intensities for all other identified proteins from the MS experiment. In order to minimize unspecific binding the pull-down experiment was optimized (Additional file 2) using 10\% glycerol in the washing step (Figure 3). The corresponding results included 1168 proteins in total, of which 20 were $\mathrm{SH} 2$-proteins with an intensity ratio of $7.8 \%$. In the full proteome data of the single cell lines (without prior enrichment with probe 1 ) the intensity ratio of $\mathrm{SH} 2$ proteins varies from $0.06 \%$ to $0.18 \%$. This shows that the enrichment with probe $\mathbf{1}$ under optimized conditions with the mixed cell lysate increases the $\mathrm{SH} 2$ protein abundance by a factor of 43 to 87 (compare Figure 3). This clearly shows that SH2-proteins have successfully been enriched by probe 1 . Since the mixed cell lysate covers only 50 out of the 110 known SH2 domain containing proteins (Additional file 3: Figure S2) [9] and low abundant proteins get diluted by mixing, it is likely that the comparably small number of identified and enriched $\mathrm{SH} 2$ proteins is an issue of the chosen cell lines, rather than an issue of the probe. Taking additionally into account that $\mathrm{SH} 2$ proteins are generally less abundant
(Additional file 3: Figure S1) underlines the value of probe 1 for $\mathrm{SH} 2$ protein enrichment. According to the data received by proteome analysis of the NCI-60 cell line panel [28] the cell line Colo205 features high SH2 protein content (Additional file 3: Figure S1). Therefore, it was chosen for further experiments. In order to confirm specific $\mathrm{SH} 2$ domain binding, pull-down experiments were performed with lysates derived from Colo205 cells treated with the phosphatase inhibitor pervanadate or water as the control (Additional file 4). The measured differences were quantified with intensity based label free quantification using the MaxQuant software. In the presence of pervanadate phosphorylated tyrosine residues should not be removed by phosphatases. Thus, SH2 domains are likely to be occupied by the excess of naturally occurring pY peptides and proteins competing with the immobilized bait. Hence, the amount of enriched $\mathrm{SH} 2$ proteins is decreased compared to the control experiment.

Although the Colo205 cell line seemed promising for further experiments, only $8 \mathrm{SH} 2$ proteins out of 23 present in the Colo205 proteome [28] were successfully quantified in three replicates. However, PI3K, SRC and

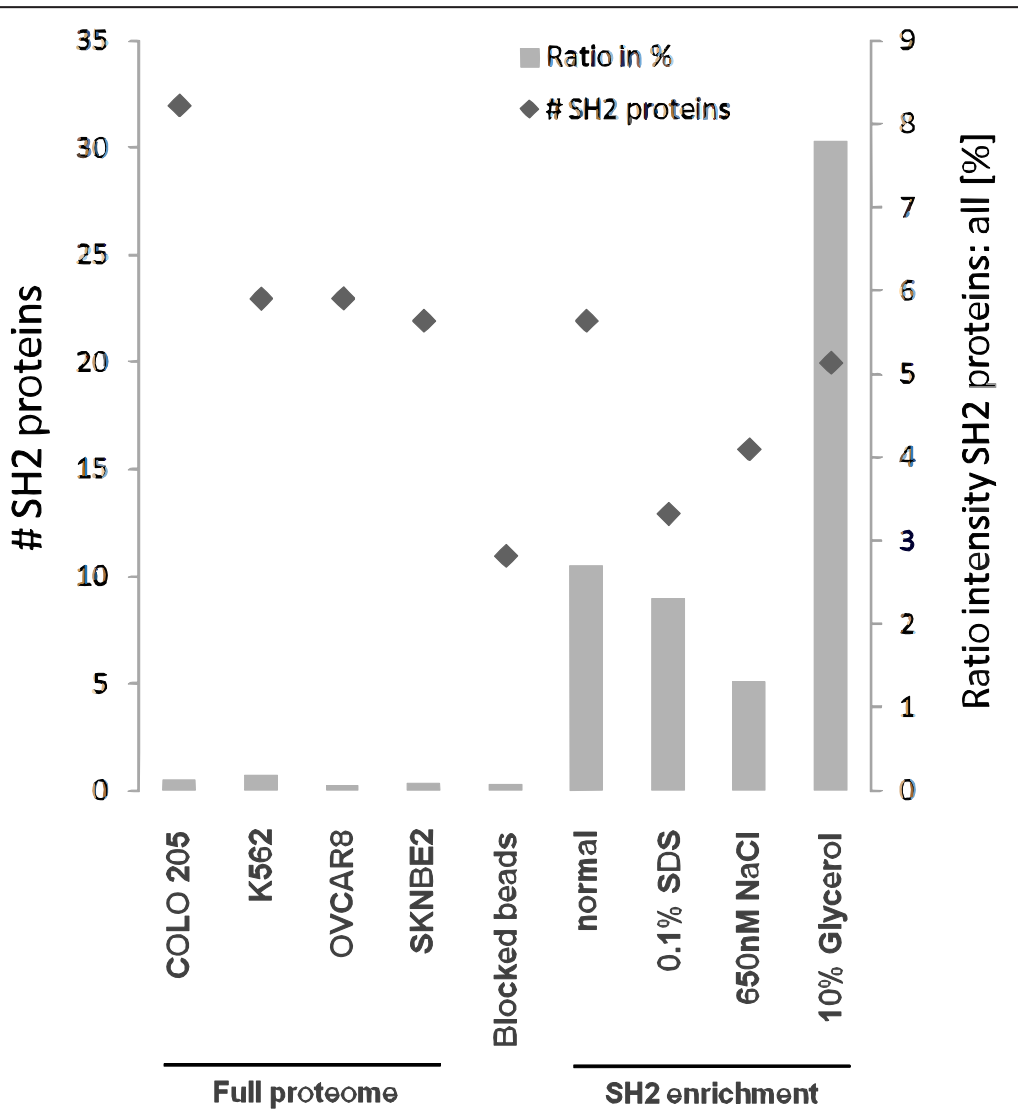

Figure $3 \mathrm{SH} 2$ protein enrichment. The MS-intensity ratio of $\mathrm{SH} 2$ proteins in relation to all identified proteins (right $y$ axis) and the number of identified SH2 proteins (left y axis). Displayed is full proteome data from Colo205, K562, Ovcar8 and SKNBE2 cells (without probe 1 enrichment), pull-down data applying blocked beads and pull-down data applying probe $\mathbf{1}$ to mixed cell lysates of Colo205, K562, Ovcar8 and SKNBE2 cells. 
ABL1 kinase that contain structurally very similar SH2 domains [29] have been reproducibly enriched in all data sets. These findings indicate that the probe molecule could be optimized for $\mathrm{SH} 2$ proteins with structurally similar SH2 domains by varying the alkyl based pY+3 binding moiety [29]. According to the "two-pronged plug and socket" mechanism these results additionally imply that the probe structure could be varied for proteins containing other $\mathrm{SH} 2$ families by incorporating a different moiety at this position. The triplicate analysis of the intensity ratios of the $\mathrm{SH} 2$ proteins shows that the amount of enriched $\mathrm{SH} 2$ proteins is significantly lower (7\%) for the pervanadate treated samples compared to $14 \%$ in the control experiment without pervanadate (compare Figure 4B). All identified SH2 proteins show a distinct difference between the pervanadate treated and the untreated conditions. This implies that the captured $\mathrm{SH} 2$ proteins bind specifically to the probe as expected. Additionally, in terms of LFQ intensities $\mathrm{SH} 2$ protein enrichment was slightly increased when using Colo205 cells only (Figure 4B) compared to the IAP experiment with mixed cell lysate (Figure 3). Although probe 1 was able to specifically enrich $\mathrm{SH} 2$ proteins and furthermore identified 22 different $\mathrm{SH} 2$ proteins, more effective $\mathrm{SH} 2$ probes are still needed to obtain complete $\mathrm{SH} 2$ protein coverage.

The PI3 kinases are targets of current research [10]. They generate lipid second messengers, act as membrane docking sites for various downstream effector proteins and consist of heterodimeric complexes of regulatory, $\mathrm{SH} 2$ domain containing subunits and catalytic subunits lacking SH2 domains. The catalytic domains $\mathrm{p} 110 \alpha$ (PI3KCA), p110 $\beta$ (PI3KCB) and $\mathrm{p} 110 \delta$ dimerize each with any of the regulatory subunits $\mathrm{p} 85 \alpha$ (PI3KR1), $\mathrm{p} 85 \beta$ (PI3KR2), p 55 $\gamma, \mathrm{p} 55 \alpha$ or $\mathrm{p} 50 \alpha$ [32]. The regulatory subunits stabilize the thermally labile catalytic subunits and inhibit their catalytic activity. Upon cellular stimulation, these heterodimers are recruited to membrane proximal pY proteins and the catalytic subunits thereby activated. The catalytic subunits PI3KCA and PI3KCB as well as the corresponding regulatory subunits (three SH2 domains: [30] C-terminal, internal and N-terminal) PI3KR1 and PI3KR2 have been enriched by probe 1 under pervanadate-free conditions. The PI3K subunits show significantly high differences between the controls and the pervanadate depleted samples. These results imply that the regulatory subunits are captured specifically by an interaction of one of their three $\mathrm{SH} 2$ domains with probe 1 . Although the catalytic subunits do not have any $\mathrm{SH} 2$ domains, they bind to the probe and show the same behavior in the pervanadate experiments. This finding shows that the catalytic subunits are captured as complexes with the regulatory subunits also under pervanadate untreated conditions. Interestingly, the involved pY residues of the catalytic PI3K subunits seem to be relatively stable towards phosphatases, since the corresponding PI3KCA/PI3KR1 heterodimer can be enriched in the IAP experiment without phosphatase inhibition (Figure 4A). It is very likely that the capturing proceeds via the $\mathrm{N}$-terminal $\mathrm{SH} 2$ domain, because this domain is not essential for the heterodimer formation and is structurally very similar to the $\mathrm{SH} 2$

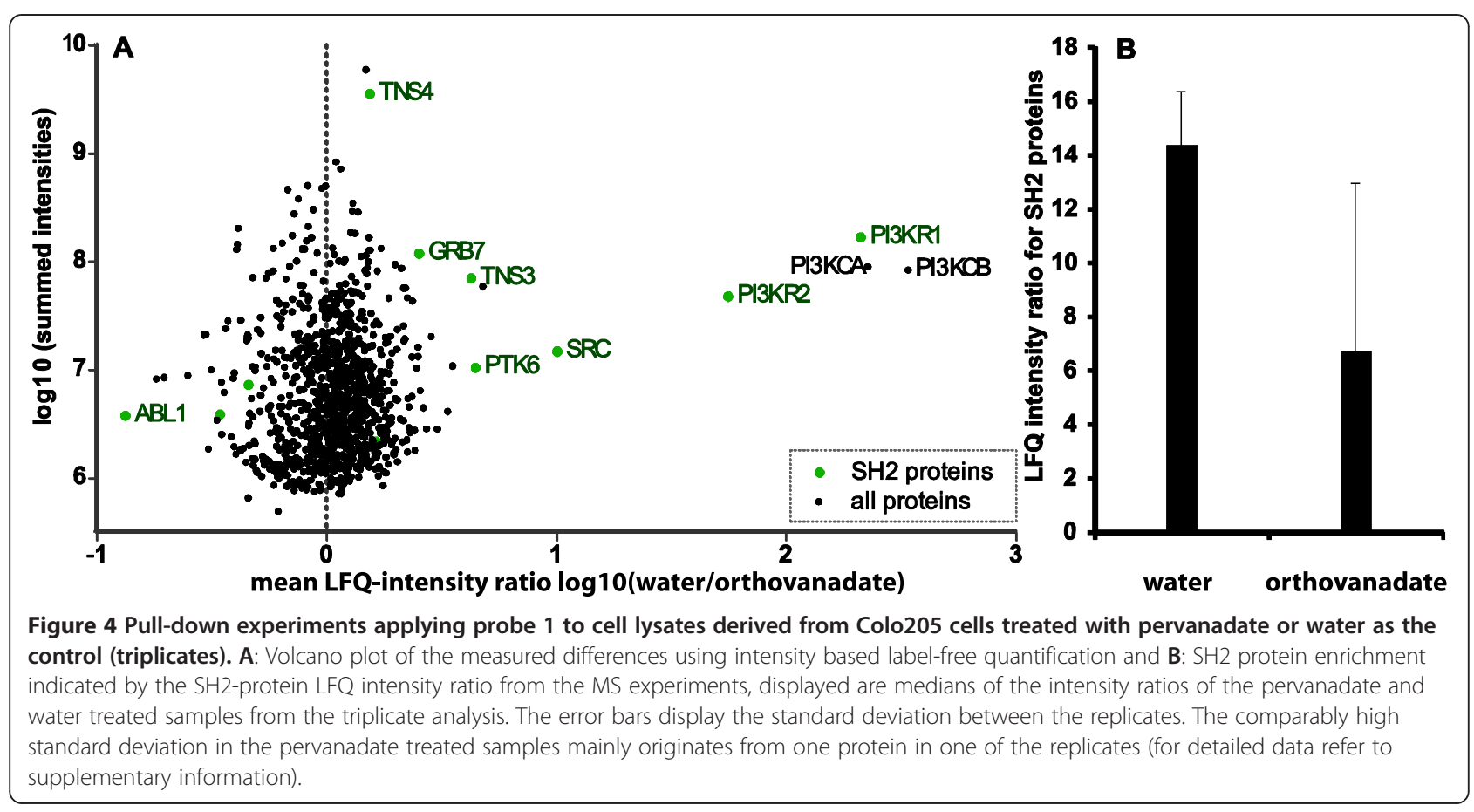


domains of SRC and ABL1 [29] that were both enriched, too. These results of the depletion experiments show, that probe $\mathbf{1}$ could be applied to evaluate $\mathrm{SH} 2$ domain addressing inhibitors of the participating regulatory subunits of PI3 kinase complexes.

Another interesting result was obtained for the kinase ABL1 that contains a SH2 domain and was highly enriched in the pervanadate experiment (Figure 4A). One possible explanation for this phenomenon could be that the $\mathrm{SH} 2$ domain is conformationally masked depending on the phosphorylation state of ABL1 and therefore not enriched under pervanadate conditions. A similar correlation was previously shown for Tyr70 and the SH3 domain of ABL1 [31]. However, further experiments are required to investigate that hypothesis.

A competitive binding experiment was performed to validate the results obtained under pervanadate-depletion conditions (Additional file 4). Dose response pull-down experiments were accomplished in Colo205 lysates with increasing concentrations of the free compound 1 that competes with the immobilized probe $\mathbf{1}$ for $\mathrm{SH} 2$ domain binding (Additional file 5). As depicted in Figure 5 probe 1 shows $\mathrm{IC}_{50}$ values in the low micromolar range (10-23 $\mu \mathrm{M})$ for the captured regulatory PI3K subunits. These values are comparable with the data published for several SH2 domain addressing inhibitors $(3-50 \mu \mathrm{M})$ $[4,33,34]$. Additionally, these findings underscore the results of the pervanadate depletion experiments that indicate that the regulatory PI3 kinase subunits specifically

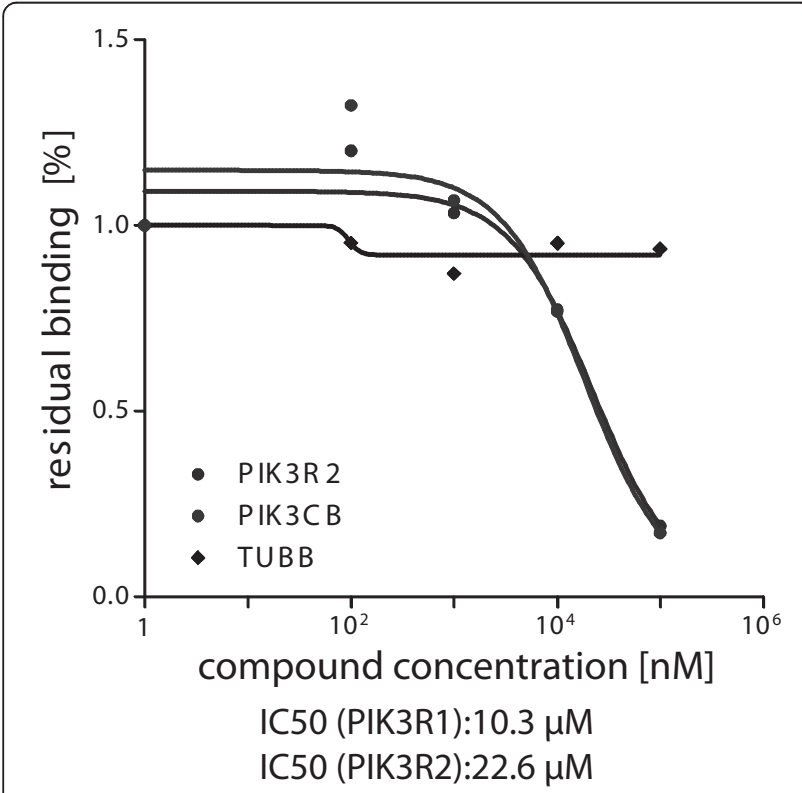

Figure 5 Dose-response curves. Pulldown experiments were conducted using probe $\mathbf{1}$ with Colo205 cell lysate and increasing concentrations of the free compound $\mathbf{1}$. interact with probe $\mathbf{1}$ via an $\mathrm{SH} 2$ domain. The regulatory PI3K subunits PI3KR1 and PI3KR2 contain a C-terminal and an $\mathrm{N}$-terminal SH2 domain. The real $\mathrm{IC}_{50}$ values are likely smaller than the measured values for PI3KR1 and PI3KR2, since it is impossible to distinguish whether one of the two domains or both simultaneously interact with equal affinity with probe 1 [35]. As expected all identified $\mathrm{SH} 2$ proteins show a dose dependent decrease in the competition experiment, whereas the other proteins such as tubulin beta chain (TBB) that is one of the most abundant proteins in the cell and in the pull-down results, do not show dose dependent decrease. These results render probe $\mathbf{1}$ a valuable tool for the investigation of PI3K subunit interactions and additionally underline the potency of probe $\mathbf{1}$ to bind SH2 domains.

\section{Conclusions}

Probe 1 selectively enriches $\mathrm{SH} 2$ proteins and allows for the identification of 22 of the 50 different $\mathrm{SH} 2$ proteins abundant in the chosen lysate with only one substance. The previously published IAP approach with the highest amount of captured SH2 proteins used 57 13-membered pY-peptides immobilized on magnetic beads and captured $45 \mathrm{SH} 2$ proteins [14]. Therefore, we strongly believe that our probe is already an improvement and should be considered as starting point for a full coverage $\mathrm{SH} 2$ protein resin. Such resin could be used for further $\mathrm{SH} 2$ inhibitor design or for the investigation of $\mathrm{SH} 2$ protein interactions. Additionally it could be applied to detect changes in the $\mathrm{SH} 2$ proteome of distinct tissues or cells. Since probe $\mathbf{1}$ captures two PI3 kinase complexes it can be applied for the investigation of the participating $\mathrm{SH} 2$ interactions and the evaluation of PI3K complex interrupting inhibitors. However, a more sufficient cell lysate for $\mathrm{SH} 2$ protein enrichment would be desirable.

\section{Methods}

Statistical analysis

A two-tailed student's t-test assuming equal variance was performed with log transformed LFQ intensities to obtain $p$-values for each protein $(\mathrm{p}=0.01$ highly significant, $\mathrm{p}=0.05$ significant).

\section{Docking experiment}

The docking calculations were performed using AutoDock Vina and AutoDock Tools [24]. All dockings were conducted as blinds using a search space that fits the whole protein structure. The figure was built using UCSF Chimera 1.7 [36].

\section{Sample preparation}

Cells were cultivated in humidified air supplemented with $5 \% \mathrm{CO}_{2}$ at $37^{\circ} \mathrm{C}$ using Roswell Park Memorial Institute 160 medium (RPMI1640) for the K562 (DSMZ, Deutsche 
Sammlung von Mikroorganismen und Zellkulturen GmbH, Braunschweig, Germany) and Colo205 (CLS, Cell Lines Service, Eppelheim, Germany) cells, Dulbecco's modified Eagle's medium (DMEM) for OVCAR8 (Anderson, Matthew L, Department of Pathology, Baylor College of Medicine, Houston, Texas) and SKNBE2 (DSMZ, Deutsche Sammlung von Mikroorganismen und Zellkulturen $\mathrm{GmbH}$, Braunschweig, Germany) cells and Iscove's modified Dulbecco's medium (IMDM) for HeLa013 cells. All media were supplemented with $10 \%$ fetal bovine serum (FBS).

For pervanadate treatment, $100 \mathrm{mM}$ sodium vanadate and $100 \mathrm{mM}$ hydrogen peroxide solution were mixed in equal amounts resulting in a $50 \mathrm{mM}$ pervanadate solution. The pervanadate solution was added to the cell culture medium to a final concentration of $100 \mu \mathrm{M}$ and incubated for $25 \mathrm{~min}$ at $37^{\circ} \mathrm{C}$.

Cells were washed with cold phosphate buffered saline (PBS) and harvested by lysis using $50 \mathrm{mM}$ Tris $/ \mathrm{HCl}$ $\mathrm{pH} 7.5,5 \%$ Glycerol, $1.5 \mathrm{mM} \mathrm{MgCl}_{2}, 150 \mathrm{mM} \mathrm{NaCl}$, $0.8 \%$ NP-40, $1 \mathrm{mM}$ dithiothreitol and $25 \mathrm{mM} \mathrm{NaF}$ with freshly added protease inhibitors and phosphatase inhibitors $(5 \times$ phosphatase inhibitor cocktail 1, Sigma-Aldrich, Munich, Germany, 5× phosphatase inhibitor cocktail 2, Sigma-Aldrich, Munich, Germany, $1 \mathrm{mM} \mathrm{Na} \mathrm{VO}_{4}$ and $20 \mathrm{nM}$ calyculin A, LC Laboratories, Woburn, MA, USA). Protein extracts were clarified by centrifugation for $20 \mathrm{~min}$ at $100,000 \times \mathrm{g}$ at $4^{\circ} \mathrm{C}$ and protein concentration was determined by the Bradford method.

\section{Compound coupling}

Probe 1 (Additional file 3) was immobilized on sepharose beads through covalent linkage using its primary amino group [12]. $1 \mathrm{ml}$ of NHS-activated sepharose (GE Healthcare, Freiburg, Germany) and the compound ( $2 \mu \mathrm{mol} / \mathrm{mL}$ beads) were equilibrated in DMSO. $15 \mu \mathrm{l}$ of triethylamine was added to start the coupling reaction and the mixture was incubated on an end-over-end shaker for 16-20 h in the dark. Free NHS-groups on the beads were blocked by adding $50 \mu \mathrm{l}$ aminoethanol and incubation on an end-over-end shaker for 16-20 h in the dark. Coupled beads were washed and stored in ethanol at $4^{\circ} \mathrm{C}$ in the dark. The coupling reaction was monitored by LC-MS.

\section{Affinity purification}

$\mathrm{SH} 2$ probe pulldowns were performed as described previously [12]. Briefly, cell lysates were diluted with equal volumes of $1 \times$ compound pulldown (CP) buffer (50 mM Tris/ $\mathrm{HCl} \mathrm{pH} \mathrm{7.5,} \mathrm{5 \%} \mathrm{glycerol,} 1.5 \mathrm{mM} \mathrm{MgCl}_{2}$, $150 \mathrm{mM} \mathrm{NaCl}, 25 \mathrm{mM} \mathrm{NaF}, 1 \mathrm{mM}$ dithiothreitol and freshly added protease inhibitors and phosphatase inhibitors (5x phosphatase inhibitor cocktail1, SigmaAldrich, Munich, Germany, $5 \times$ phosphatase inhibitor cocktail 2, Sigma-Aldrich, Munich, Germany, $1 \mathrm{mM}$ sodium pervanadate and $20 \mathrm{nM}$ calyculin A, LC Laboratories, Woburn, MA, USA)). For the dose response experiment, lysates were pre-incubated with the free compound for $30 \mathrm{~min}$ at $4^{\circ} \mathrm{C}$. Lysates were incubated with beads for $30 \mathrm{~min}$ at $4^{\circ} \mathrm{C}$. Subsequently, the beads were washed with $1 \times \mathrm{CP}$ buffers containing $0.4 \%$ and $0.2 \%$ NP40 and $10 \%$ glycerol. Bound proteins were eluted with $2 \times$ NuPAGE LDS Sample Buffer (Invitrogen, Darmstadt, Germany) and eluates were reduced and alkylated by $50 \mathrm{mM}$ dithiothreitol and $55 \mathrm{mM}$ chloroacetamide. Samples were then run into a 4-12\% NuPAGE gel (Invitrogen, Darmstadt, Germany) for about $0.5 \mathrm{~cm}$ to concentrate the sample prior to in-gel tryptic digestion. In-gel trypsin digestion was performed according to standard procedures.

\section{LC-MS/MS measurements}

Nanoflow LC-MS/MS was performed by coupling an Eksigent nanoLC-Ultra 1D + (Eksigent, Dublin, CA) to a LTQ-Orbitrap XL ETD (Thermo Scientific, Bremen, Germany). Peptides were delivered to a trap column $\left(100 \mu \mathrm{m} \times 2 \mathrm{~cm}\right.$, packed in-house with Reprosil-Pur $\mathrm{C}_{18^{-}}$ AQ $5 \mu \mathrm{m}$ resin, Dr. Maisch, Ammerbuch, Germany) at a flow rate of $5 \mu \mathrm{L} / \mathrm{min}$ in $100 \%$ solvent A $(0.1 \%$ formic acid in HPLC grade water). After 10 min of loading and washing, peptides were transferred to an analytical column $(75 \mu \mathrm{m} \times 40 \mathrm{~cm}$, packed in-house with ReprosilPur $\mathrm{C}_{18}$-GOLD, $3 \mu \mathrm{m}$ resin, Dr. Maisch, Ammerbuch, Germany) and separated using a $210 \mathrm{~min}$ gradient from $4 \%$ to $32 \%$ of solvent B $(0.1 \%$ formic acid, $5 \%$ DMSO in acetonitrile; solvent A: $0.1 \%$ formic acid, $5 \%$ DMSO in water) at $300 \mathrm{~nL} /$ minute flow rate. The LTQ Orbitrap XL was operated in data dependent mode, automatically switching between MS and MS [2]. Full scan MS spectra were acquired in the Orbitrap at 60,000 (m/z 400) resolution after accumulation to a target value of $1,000,000$. Tandem mass spectra were generated for up to eight peptide precursors in the linear ion trap by using collision-induced dissociation at a normalized collision energy of $35 \%$ after accumulation to a target value of 5,000 for max $100 \mathrm{~ms}$.

\section{Peptide and protein identification and quantification}

Raw MS data were processed by MaxQuant (v1.4.0.5) for peak detection and quantification [37] MS/MS spectra were searched against the UniProtKB human database (22.07.13, 88.354 sequences) using the Andromeda search engine [38] with the following search parameters: full tryptic specificity, re-quantification and match-betweenruns option, up to two missed cleavage sites, carbamidomethylation of cystein residues was set as a fixed modification and N-terminal protein acetylation, methionine oxidation and phosphorylation as variable 
modifications. Mass spectra were re-calibrated within MaxQuant (first search $20 \mathrm{ppm}$ precursor tolerance) and subsequently re-searched with a mass tolerance of $10 \mathrm{ppm}$. Fragment ion mass tolerance was set to $0.5 \mathrm{Da}$. Search results were filtered to a maximum false discovery rate (FDR) of 0.01 for proteins and peptides and a peptide length of at least 7 amino acids was required.

LFQ intensities of 0 (in control replicates) were replaced with the $1 \%$ percentile of the LFQ intensities of this respective measurement for data visualization.

\section{Additional files}

Additional file 1: Proteomic data. List of captured $\mathrm{SH} 2$ proteins.

Additional file 2: Proteomic data. Pull-down optimization.

Additional file 3: Detailed experimental procedures. Experimenta procedures and analytical data of the compound synthesis and supplementary figures and tables.

Additional file 4: Proteomic data. Data set of the pervanadate depletion experiments.

Additional file 5: Proteomic data. Data set of the dose response experiments.

\section{Abbreviations \\ ATP: Adenosine triphosphate; SRC: Proto-oncogene tyrosine-protein kinase SRC; SH2: SRC homology 2; AKT: Serine/threonine-protein kinase AKT; NF-KB: Nuclear factor kappa-light-chain-enhancer of activated B cells; JNK: c-Jun N-terminal kinase; PI3K: Phosphoinositide 3-kinase; GST: Glutathione-S-transferase; IAP: Inhibitor affinity purification; EGFR: Epidermal growth factor receptor; PI3KCA/B: Phosphoinositide 3-kinase catalytic subunit A/B; PI3KR1/2: Phosphoinositide 3-kinase regulatory subunit 1/2; $A B L 1$ : Abelson murine leukemia viral oncogene homolog 1; LFQ: Label free quantification; $I_{50}$ : Half maximal inhibitory concentration; LC-MS/MS: Liquid chromatography tandem mass spectrometry.}

\section{Competing interests}

The authors declare that they have no competing interests.

\section{Authors' contributions}

The manuscript was written through contributions of all authors. All authors read and approved the final manuscript.

\section{Acknowledgements}

We acknowledge support of the publication fee by Deutsche

Forschungsgemeinschaft and the Open Access Publication Funds of Bielefeld University.

\section{Author details}

${ }^{1}$ Organic and Bioorganic Chemistry, Department of Chemistry, Bielefeld University, Universitätsstraße 25, 33615 Bielefeld, Germany. ${ }^{2}$ Chair for Proteomics and Bioanalytics, Center of Life and Food Sciences Weihenstephan, Technische Universität München, Freising, Germany. ${ }^{3}$ German Cancer Consortium (DKTK), Munich, Germany. ${ }^{4}$ German Cancer Research Center (DKFZ), Heidelberg, Germany. ${ }^{5}$ Center for Integrated Protein Science Munich, Freising, Germany.

Received: 19 February 2014 Accepted: 9 July 2014

Published: 16 July 2014

\section{References}

1. Cohen P: Protein kinases - the major drug targets of the twenty-first century? Nat Rev Drug Discov 2002, 1:309-315.
2. Parsons SJ, Parsons JT: Src family kinases, key regulators of signal transduction. Oncogene 2004, 23:7906-7909.

3. Noble MEM: Protein kinase inhibitors: insights into drug design from structure. Science 2004, 303:1800-1805.

4. Page BDG: Small molecule STAT5-SH2 domain inhibitors exhibit potent antileukemia activity. J Med Chem 2012, 55:1047-1055.

5. $\mathrm{Vu} C \mathrm{CB}$ : Recent advances in the design and synthesis of $\mathrm{SH} 2$ inhibitors of Src, Grb2 and ZAP-70. Curr Med Chem 2000, 7:1081-1100.

6. Zhang J, Yang PL, Gray NS: Targeting cancer with small molecule kinase inhibitors. Nat Rev Cancer 2009, 9:28-39.

7. Zhang Y, Zhang J, Yuan C, Hard RL, Park I-H, Li C, Bell C, Pei D: Simultaneous binding of two peptidyl ligands by a Src homology 2 domain. Biochemistry 2011, 50:7637-7646.

8. Uniprot Consortium: Update on activities at the Universal Protein Resource (UniProt) in 2013. Nucleic Acids Res 2012, 41:D43.

9. Liu BA, Jablonowski K, Raina M, Arcé M, Pawson T, Nash PD: The human and mouse complement of $\mathrm{SH} 2$ domain proteins-establishing the boundaries of phosphotyrosine signaling. Mol Cell 2006, 22:851-868.

10. Akinleye A, Avvaru P, Furqan M, Song Y, Liu D: Phosphatidylinositol 3-kinase (PI3K) inhibitors as cancer therapeutics. J Hematol Oncol 2013, 6:88.

11. Kraskouskaya D, Duodu E, Arpin CC, Gunning PT: Progress towards the development of SH2 domain inhibitors. Chem Soc Rev 2013, 42:3337.

12. Bantscheff M, Eberhard D, Abraham Y, Bastuck S, Boesche M, Hobson S, Mathieson T, Perrin J, Raida M, Rau C, Reader V, Sweetman G, Bauer A, Bouwmeester T, Hopf C, Kruse U, Neubauer G, Ramsden N, Rick J, Kuster B, Drewes G: Quantitative chemical proteomics reveals mechanisms of action of clinical ABL kinase inhibitors. Nat Biotechnol 2007, 25:1035-1044.

13. Wiśniewski JR, Zougman A, Nagaraj N, Mann M: Universal sample preparation method for proteome analysis. Nat Meth 2009, 6:359-362.

14. Tinti M, Kiemer L, Costa S, Miller ML, Sacco F, Olsen JV, Carducci M, Paoluzi S, Langone F, Workman CT, Blom N, Machida K, Thompson CM, Schutkowski M, Brunak S, Mann M, Mayer BJ, Castagnoli L, Cesareni G: The SH2 domain interaction landscape. Cell Reports 2013, 3:1293-1305.

15. Blagoev B, Ong S-E, Kratchmarova I, Mann M: Temporal analysis of phosphotyrosine-dependent signaling networks by quantitative proteomics. Nat Biotechnol 2004, 22:1139-1145.

16. Schulze WX, Deng L, Mann M: Phosphotyrosine interactome of the ErbB-receptor kinase family. Mol Syst Biol 2005, 1:E1-E13.

17. Lemeer S, Bluwstein A, Wu Z, Leberfinger J, Müller K, Kramer K, Kuster B: Phosphotyrosine mediated protein interactions of the discoidin domain receptor 1. J Proteomics 2012, 75:3465-3477.

18. Charifson PS: Peptide ligands of pp60c-src SH2 domains: a thermodynamic and structural study. Biochemistry 1997, 36:6283-6293.

19. Schirle M, Bantscheff M, Kuster B: Mass spectrometry-based proteomics in preclinical drug discovery. Chem Biol 2012, 19:72-84.

20. Gay B, Suarez S, Caravatti G, Furet P, Meyer T, Schoepfer J: Selective GRB2 SH2 inhibitors as anti-Ras therapy. Int J Cancer 1999, 83:235-241.

21. Tong L, Warren TC, King J, Betageri R, Rose J, Jakes S: Crystal structures of the human p56lck SH2 domain in complex with two short phosphotyrosyl peptides at 1.0 Å and 1.8 Å resolution. J Med Bio/ 1996, 256:601-610.

22. Imhof D: Sequence specificity of SHP-1 and SHP-2 Src homology 2 domains: critical roles of residues beyond the $\mathrm{pY}+3$ position. J Biol Chem 2006, 281:20271-20282.

23. Quartararo JS, Wu P, Kritzer JA: Peptide bicycles that inhibit the Grb2 SH2 domain. ChemBioChem 2012, 13:1490-1496.

24. Trott O, Olson AJ: AutoDock Vina: improving the speed and accuracy of docking with a new scoring function, efficient optimization, and multithreading. J Comput Chem 2009, 31:455-461.

25. Zhou S, Shoelson SE, Chaudhuri M, Gish G, Pawson T, Haser WG, King F, Roberts T, Ratnofsky S, Lechleider RJ, Neel BG, Birge RB, Fajardo JE, Chou MM, Hanafusa H, Schaffhausen B, Cantley LC: SH2 domains recognize specific phosphopeptide sequences. Cell Reports 1993, 72:767-778.

26. Yokomatsu T, Murano T, Suemune K, Shibuya S: Facile synthesis of aryl (difluoromethyl)phosphonates through CuBr-mediated cross coupling reactions of [(diethoxyphosphinyl)difluoromethyl]zinc bromide with aryl iodides. Tetrahedron 1997, 53:815-822.

27. Qabar MN, Urban J, Kahn M: A facile solution and solid phase synthesis of phosphotyrosine mimetic l-4-[diethylphosphono(difluoromethyl)]- 
phenylalanine $\left(\mathrm{F}_{2} \mathrm{Pmp}(\mathrm{EtO})_{2}\right)$ derivatives. Tetrahedron 1997,

53:11171-11178.

28. Gholami AM, Hahne H, Wu Z, Auer FJ, Meng C, Wilhelm M, Kuster B: Global proteome analysis of the NCl-60 cell line panel. Cell Rep 2013, 4:609-620.

29. Waksman G, Shoelson SE, Pant N, Cowburn D, Kuriyan J: Binding of a high affinity phosphotyrosyl peptide to the $\mathrm{Src} 5 \mathrm{SH} 26$ domain: crystal structures of the complexed and peptide-free forms. Cell 1993, 72:779-790.

30. Miled N, Yan Y, Hon W-C, Perisic O, Zvelebil M, Inbar Y, Schneidman-Duhovny D, Wolfson HJ, Backer JM, Williams RL: Mechanism of two classes of cancer mutations in the phosphoinositide 3-kinase catalytic subunit. Science 2007, 317:239-242.

31. Nagar B, Hantschel O, Seeliger M, Davies JM, Weis WI, Superti-Furga G, Kuriyan J: Organization of the $\mathrm{SH} 3-\mathrm{SH} 2$ unit in active and inactive forms of the c-Abl tyrosine kinase. Mol Cell 2006, 21:787-798.

32. Geering B, Cutillas PR, Nock G, Gharbi SI, Vanhaesebroeck B: Class IA phosphoinositide 3-kinases are obligate p85-p110 heterodimers. Proc Natl Acad Sci U S A 2007, 114:7809-7814.

33. Günther UL, Liu Y, Sanford D, Bachovchin WW, Schaffhausen B: NMR analysis of interactions of a phosphatidylinositol 3'-kinase SH2 domain with phosphotyrosine peptides reveals interdependence of major binding sites. Biochemistry 1996, 35:15570-15581.

34. Stankovic CJ, Surendran N, Lunney EA, Plummer MS, Para KS, Shahripour A, Fergus JH, Marks JS, Herrera R, Hubbell SE, Humblet C, Saltiel AR, Stewart BH, Sawyer TK: The role of 4-phosphonodifluoromethyl- and 4-phosphono-phenylalanine in the selectivity and cellular uptake of 5SH26 domain ligands. Bioorg Med Chem Lett 1997, 7:1909-1914.

35. O'Brien R, Rugman P, Renzoni D, Layton M, Handa R, Hilyard K, Waterfield $M D$, Driscoll PC, Ladbury JE: Alternative modes of binding of proteins with tandem SH2 domains. Protein Sci 2000, 9:570-579.

36. Pettersen EF, Goddard TD, Huang CC, Couch GS, Greenblatt DM, Meng EC, Ferrin TE: UCSF Chimera? A visualization system for exploratory research and analysis. J Comput Chem 2004, 25:1605-1612.

37. Cox J, Mann M: MaxQuant enables high peptide identification rates, individualized p.p.b.-range mass accuracies and proteome-wide protein quantification. Nat Biotechnol 2008, 26:1367-1372.

38. Cox J, Neuhauser N, Michalski A, Scheltema RA, Olsen JV, Mann M: Andromeda: a peptide search engine integrated into the MaxQuant environment. J Proteome Res 2011, 10:1794-1805.

doi:10.1186/1477-5956-12-41

Cite this article as: Höfener et al:: Probing SH2-domains using Inhibitor Affinity Purification (IAP). Proteome Science 2014 12:41.

\section{Submit your next manuscript to BioMed Central and take full advantage of:}

- Convenient online submission

- Thorough peer review

- No space constraints or color figure charges

- Immediate publication on acceptance

- Inclusion in PubMed, CAS, Scopus and Google Scholar

- Research which is freely available for redistribution 